Article

\title{
Comparative Proteomic Profiling between Each of Two Consecutive Developmental Stages of the Solanum Fruit Fly, Bactrocera latifrons (Hendel)
}

\author{
Chiou Ling Chang * and Scott M. Geib \\ USDA, Agricultural Research Service, Daniel K. Inouye U.S. Pacific Basin Agricultural Research Center, \\ 64 Nowelo Street, Hilo, HI 96720, USA; scott.geib@ars.usda.gov \\ * Correspondence: stella.chang@ars.usda.gov; Tel: +1-808-959-4312
}

Received: 11 May 2018; Accepted: 2 July 2018; Published: 9 July 2018

\begin{abstract}
The Solanum fruit fly, Bactrocera latifrons (Hendel), has a complex life cycle including multiple stages (egg, larva, pupa, and adult). Understanding the details of "what", "when", "where", "why", and "how" many hundred thousand proteins operate in this insect, interact, and express between each two consecutive developmental stages at molecular level not only can expand our knowledge, but also lead to the development of novel fruit fly control techniques. We tried to find what, when, and where in this study. Why and how will be presented in upcoming papers. We conducted a proteome profiling using 2-D gel electrophoresis and mass spectrometry. Samples of 3-day-old eggs, 1- and 10-day-old larvae, 1- and 10-day-old pupae, 1- and 9-day-old females and males of $B$. latifrons were used. A custom peptide database, derived from the de novo B. latifrons whole genome assembly was used for peptide identification. Differentially expressed proteins (DEPs) with significant fold expression and protein functions between two consecutive developmental stages were identified, annotated, described, and listed in gel images and/or charts. With this foundational information, we are not only providing valuable information, but also any impacts due to the biotic or abiotic environmental factors can be identified and manipulated, and lead to further research on gene editing and biomarker discovery.
\end{abstract}

Keywords: Bactrocera latifrons; proteomic analysis; developmental biology; life cycle

\section{Introduction}

New tools for fruit fly control using gene editing will be an important supplement to current chemical treatments, sterile insect techniques, and biological control. To initiate gene editing research, specific target genes must be identified and validated. There were ways to identify and validate target genes/proteins using genomic and transcriptomic approach. Proteomics has expanded the research and development of targets in some insects such as Drosophila, mosquito, aphid, honey bee, silkworm, and Helicoverpa armigera, especially expression proteomics that has been used for identification and quantification or expression level of proteins of the cell, tissue, organism at different developmental stages or at different environmental conditions [1]. However, changes on the proteome of the Solanum fruit fly depending on the developmental stage has not yet been reported.

Tephritids such as the solanum fruit fly, Bactrocera latifrons (Hendel) are native to South and Southeast Asia and distributed among China, Taiwan, Malaysia, Thailand, Laos, India, Pakistan, Tanzania, Kenya, and Hawaii [2]. It infests Solanancease and Cucurbitaceae crops and has the potential to permanently establish itself, and compete, and/or coexist with other tephritid fruit fly species [3]. Morphological and ecological characteristics have been described [3-5]. There are four stages (egg, larva, pupa, and adult) in its life cycle ( 48 days), 21 days from egg to adult; 
2-3 days for the eggs to hatch, 8-9 days for larval development, adult emergence in 10 days, and pre-oviposition period is 10-11 days. While general biological data are available, information on protein expression, which may point to key target genes useful for genetic control programs, is lacking. Current proteomics technologies not only identify protein expression, but also post-translation modifications and interactions among different insect proteins. Future studies are needed to compare proteomic results across taxonomic groups and life stages, leading to advances in our understanding of insect proteomics [1].

Understanding basic fruit fly molecular biology at each developmental life stage in their life cycle to learn "what" these differentially expressed proteins within each developmental stage and between each two subsequent developmental stages are, and when and where they express, has been the most important element for improving the quality of mass-rearing fruit flies for Sterilization Insect Technique (SIT). This information can be used as foundational/baseline information to later manipulate or identify the causes/pathway from any biotic or abiotic influences to explain why and how they express using gene editing techniques. Here, we descriptively report on proteomic profiles through development between each two consecutive stages from an egg to an adult of the Solanum fruit fly using proteomic approach for the first time.

\section{Results and Discussion}

This report discusses all the subsequent stages-egg (E) to larva (L), larva to pupa (P), pupa to male (M), pupa to female (F), 1-day-old male and female, and 9-day-old male and female.

Solanum fruit flies undergo three stages of development—egg, larva, and pupa—before emerging as adults. Under natural field conditions, approximately 9-587 eggs are laid below the skin of the host fruit. Eggs hatch within 2-3 days, and the larvae feed for about 8.5 days before pupation. Pupation occurred in the soil for about 10.2 days. Adults occur all year around and females start oviposition at 6-17 days and continue laying eggs for as long as 117 days [2]. In our observations under laboratory conditions, eggs hatch after three days, larvae feed for about 10 days, and pupae take 12 days to develop into adults. In this study, we aimed to evaluate the protein changes in lab-reared flies between 3-day-old eggs (E3) and 1-day-old larvae (L1), 10-day-old larvae (L10) and 1-day-old pupae (P1), 10-day-old pupae (P10) and 1-day-old females (F1), and 10-d-old pupae (P10) and 1-day-old males (M1).

During development, insects undergo considerable cellular remodeling requiring the combined actions of thousands of proteins. In this study, the proteome profiling was evaluated based on the comparison between sequential stages. Generally speaking, the pupal stage expressed the most number of significantly differentially expressed proteins. While the pupal stage was most differentiated from other stages in terms of protein expression, the egg stage showed the least changes in protein expression compared with the others. The descriptions below are based on development between two consecutive stages:

\subsection{Eggs (E3)-Larvae (L1)}

Protein changes in developmental process from eggs to larvae were the most significant changes of the stage. Between 3-day-old egg (E3) to newly hatched 1-day-old larvae (L1), there were a total of 70 significantly differentially expressed protein spots (Figure 1A,B). Among all, 41 protein spots containing 10 proteins were expressed as down-regulated by 51 to $96 \%(\mathrm{~L} 1 / \mathrm{E} 3$ ratio $=0.04-0.49)$ (Figure 1B) whereas 29 protein spots containing 24 proteins were expressed as up-regulated by 2.01 to 34.34-fold (Table 1; Figure 1A)). 41 down-regulated protein spots were composed of 10 proteins including maltase 2 (7), transferrin (5), vitellogenin-1 (12), vitellogenin-2 (7), peroxiredoxin 1 (1), peroxiredoxin $5(1)$, protein amalgam (1), peptidyl-prolyl cis-trans isomerase $6(1)$, heat shock protein 23 (5), and heat shock protein 83 (1). Numbers inside parentheses following the protein names were the number of spots that belong to that same protein. Vitellogenin is the precursor of the lipoproteins and phosphoproteins that make up most of the protein content of yolk. At this 
moment, the embryogenesis is close to the end; therefore, both vitellogenin- 1 and vitellogenin-2 were greatly downregulated by $64-93 \%$ and $54-89 \%$, respectively. Heat shock protein 23 and 83 were decreased by 77-92\%, and transferrin and maltase 2 were reduced by $76-96 \%$ and $56-95 \%$, respectively. Protein amalgam (Ama) was downregulated 68\%. Protein Ama (a secreted neuronal adhesion protein) contains three tandem immunoglobulin domains. It has both homophilic and heterophilic cell adhesion properties during early stages of Drosophila development and is required for axon guidance and fasciculation. The function of this protein remains elusive [6,7]. Peroxiredoxin 1 (Prx1) was downregulated by $73 \%$. Peroxiredoxin 1 is involved in redox regulation of the cell. It is a molecular chaperone that acts as a regulator of hydrogen peroxide signaling. Downregulated Prx1 indicates the increase of survival (www.roswellpark.org/commercialization). It may play an important role in eliminating peroxides generated during metabolism by reducing peroxides through the thioredoxin system. It might participate in the signaling cascades of growth factors and tumor necrosis factor-alpha by regulating the intracellular concentrations of $\mathrm{H}_{2} \mathrm{O}_{2}$. It reduces an intramolecular disulfide bond in GDPD5 that gates the ability to GDPD5 to drive post-mitotic motor neuron differentiation. Ubiquitin-related modifier 1 (Urm1) is a ubiquitin-like molecule (UBL) with the dual capacity to act both as a sulphur carrier and posttranslational protein modifier. In the Drosophila melanogaster, Urm1 (CG33276) and its E1 activating enzyme Uba4 (CG13090) functioned together to induce protein urmylation in vivo. Urm1 conjugated to target proteins in general. Peroxiredoxin 5 (Prx5) is dependent on Uba4. A complete loss of Urm1 is lethal in flies, although a small number of adult zygotic Urm1 (n123) mutant escapers can be recovered. These escapers display a decreased general fitness and shortened lifespan, but they are resistant to oxidative stress. Therefore, Urm1 is a UBL that is involved in the regulation of JNK signaling and the response against oxidative stress in the fruit fly [8]. In this study, peroxiredoxin 5 (0.49) and Peptidyl-prolyl cis-trans isomerase 6 (0.46) did not show any significant changes between egg to larval stage. Heat shock protein 83 (hsp83) was downregulated by $75 \%$. Hsp 83 is a molecular chaperone that is involved in cell cycle control and signal transduction to promote the maturation, structural maintenance, and proper regulation of specific target proteins. Together with Hop (heat shot organizing protein) and piwi (regulatory proteins responsible for stem cell and germ cell differentiation), hsp83 mediates canalization, also known as developmental robustness. In Ceratitis capitate, Cchsp83 RNA expression is highly regulated during embryonic development, but the temporal fluctuations in RNA levels during embryogenesis were not followed by similar fluctuations in the levels of the protein [9]. Our results in B. latifrons confirmed this finding.

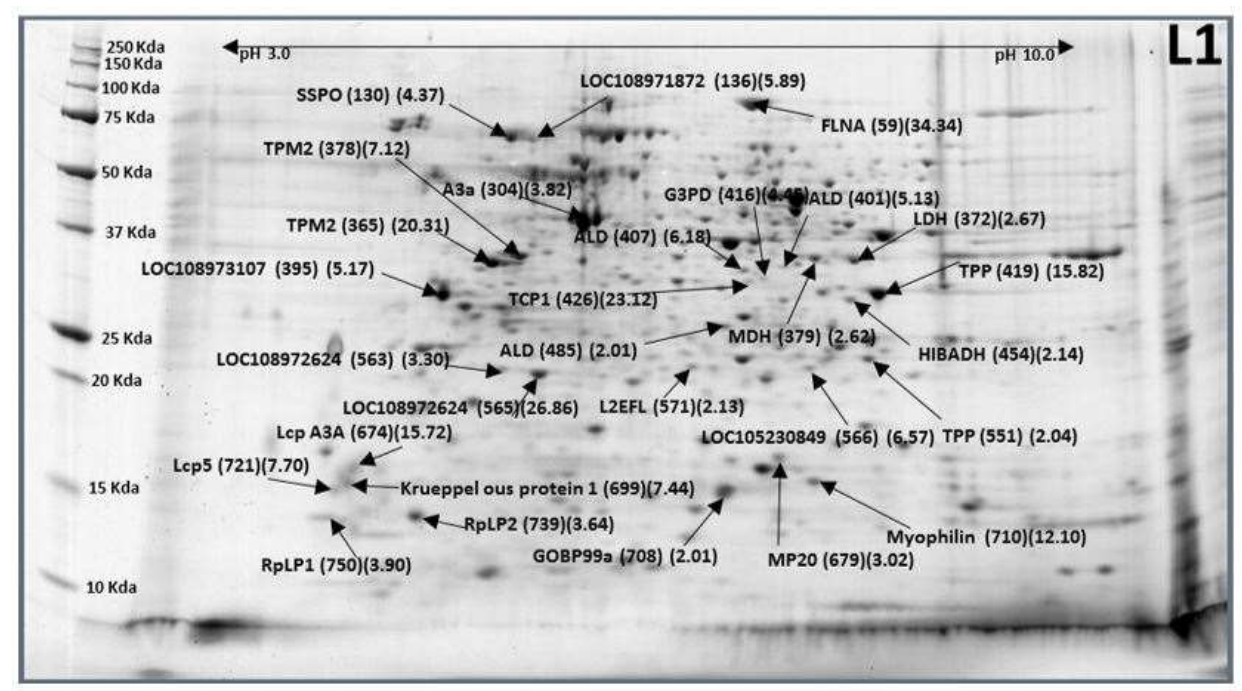

(A)

Figure 1. Cont. 


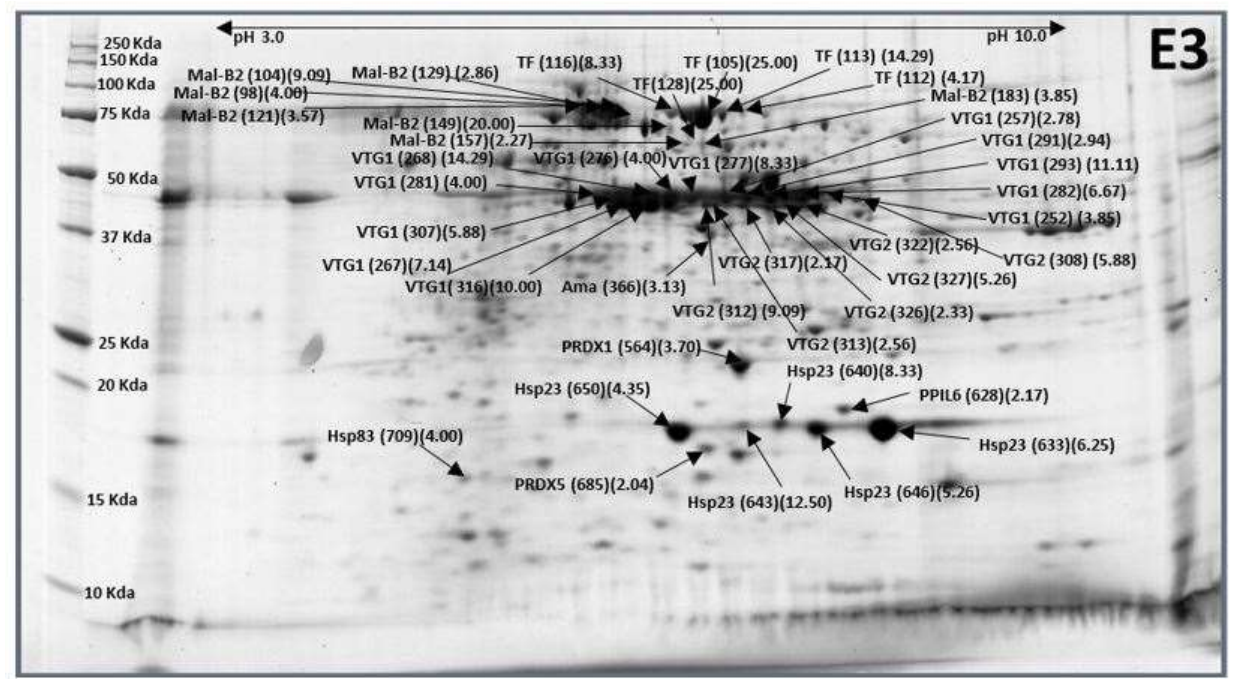

(B)

Figure 1. Representative images of 1-day-old larvae (A) and 3-day-old egg (B) of Bactrocera latifrons. Images were labelled with protein names (spot ID\#) (L1/E3 ratios).

Twenty-four up-regulated proteins from 29 spots (Figure 1B) were SCO-spondin, Uncharacterized protein LOC108971872 isoform X2, Actin-2, muscle-specific, tropomyosin-2 (2), L-lactate dehydrogenase, malate dehydrogenase, Uncharacterized protein LOC108973107 isoform X1, fructose-bisphosphate aldolase (3), glyceraldehyde-3-phosphate dehydrogenase, trehalose-phosphate phosphatase (2), T-complex protein 1 subunit gamma, putative 3-hydroxyisobutyrate dehydrogenase MC, Uncharacterized protein LOC108972624 (2), Uncharacterized protein LOC105230849, protein lethal (2) essential for life, filamin-A, Larval cuticle protein A3A, muscle-specific protein 20, krueppelous protein 1, general odorant-binding protein 99a, myophilin, larval cuticle protein 5, 60S acidic ribosomal protein P2, and $60 \mathrm{~S}$ acidic ribosomal protein P1 (Table 1). Again, abovementioned numbers inside parentheses following the protein names represented the number of spots that belong to that same protein. SCO-spondin is a large glycoprotein with a multi-domain organization that is secreted early by the developing central nervous system. It is a crucial embryonic cerebrospinal fluid (eCSF) factor that has been shown to regulate the balance between proliferation and differentiation of the brain neuroepithelial cells [10]. SCO-spondin was upregulated by 4.37-fold at 1-day-old larvae. Both $H$. armigera $\mathrm{A} 3 \mathrm{a}$ and $\mathrm{A} 3 \mathrm{~b}$ genes are expressed during pupal development and in the brain of newly enclosed adults based on polymerase chain reaction and northern blot analysis [11]. Muscle-specific Actin-2 was upregulated by 3.82-fold in the larvae in this study, which added a piece of information that is expressed in the larvae stage. The function of tropomyosin- 2 was dictated as a fundamental step of myogenesis prior to regulating contraction in the sarcomere [12]. Myogenesis is the formation of muscular tissue, particularly during embryonic development. Our result with tropomyosin-2 upregulated by 7-20-fold in L1 larval stage seems to confirm their report. Proteins that involve glycolysis such as lactate dehydrogenase, malate dehydrogenase, fructose-bisphosphate aldolase, glyceraldhyde-3-phosphate dehydrogenase, 3-hydrozyisobutyrate dehydrogenase, and trehalose-phosphate phosphatase were all expressed upregulated. Trehalose phosphatephosphatase (TPP) converts trehalose-6-phosphate to free trehalose. Trehalose can protect cells from desiccation, dehydration, heat, cold, and oxidation by decreasing protein denaturation through protein-trehalose interactions [13]. T-complex protein 1 subunit gamma (TCP-1) was found to interact with actin and tubulin proteins, suggesting that the complex may have a role in maintaining the structural dynamics of the cytoskeleton of parasites $[14,15]$. In this transformation, TCP-1 was increased by 23-fold to support the cytoskeleton. Protein lethal (2) essential for life [L(2)efl] is a small heat-shock (HS)-homologous gene, identified on the right arm of the second chromosome at locus 59F4,5. It acts as 
a regulator of lateral transverse muscle shapes in Drosophila melanogaster. It was expressed in embryonic, larval, and adult somatic and heart muscles during Drosophila development [16]. L(2)efl protein has demonstrated muscle-specific expression in late embryos and accumulates in a dotty pattern close to the muscle cell membrane. Our results confirmed their finding [17]. Filamin-A is a key component of a versatile signaling scaffold complex [18-22] that anchors various transmembrane proteins to the actin cytoskeleton and provides a scaffold for a wide range of cytoplasmic and nuclear signaling proteins, andwas increased by 34 -fold during this transformation from eggs to larvae. Uncharacterized protein LOC108972624 was increased by 26.86-fold. Muscle-specific protein 20 (Mp20) that is not detected in the asynchronous oscillatory flight muscles in D. melanogaster, but is found in most, if not all, other muscles (the synchronous muscles) [23]. Mp20 was upregulated 3-fold in L1 stage. Krueppelous protein 1 Myophilin RNA is first expressed late in embryogenesis at stage 15 in Drosophila melanogaster. It confirmed our finding that at 1st day of larvae, myophilin was increased by 12 -fold. We speculated that it is necessary for filament assembly in all muscles, and flightin for stability of flight muscle thick filaments in adult flies [24].

\subsection{Larvae (10-Day-Old, L10)-Pupae (1-Day-Old, P1)}

Protein changes between last day of larvae (L10) and 1st day pupae (P1) were 19 protein spots containing 17 proteins expressing down-regulated by 50 to 95\% (P1/L10 ratio $=0.05-0.50)$ (Figure 2A) whereas 19 protein spots contain only 12 proteins expressing up-regulated by 2.30 to 11.17 -fold, mainly cuticle proteins (Table 2 and Figure 2B). Among these differentially expressed proteins, cuticle protein changes the most. Larval cuticle protein 5 (Lcp5) up-regulated by 3.29, 3.94, 7.26)-fold, cuticular protein 47Eg (CP47Eg) by 5.84-fold, uncharacterized protein LOC108972251 by $17.48(6.31,11.17)$-fold, and LOC108969313 by 4.7-fold. Other downregulated uncharacterized proteins (including LOC108971874, LOC108971872, LOC108973107, 108969313, and LOC108972625) were down-regulated by 0.29-0.4, enolase-phosphatase E1 by 4.21, Tropomyosin-2 (TPM2) up-regulated by 8.97 (2.51, 2.75, 3.71)-fold while muscle-specific 20 (MP20) and myophilin down-regulated by 70-95\%. Regucalcin (RGN) up-regulated by 10 -fold. Calreticulin (CALR) was down-regulated by $90 \%$. Protein yellow was upregulated by 3.64-fold in the transition from L10 to P1 stage. Protein yellow is only found in insects. In Drosophila, it is involved in pigmentation and male mating behavior, though the mechanism by which it influences melanization is not clear. SCO-spondin was down-regulated a little. In vitro, SCO-spondin was reported as a promotor of neuronal survival and differentiation, but its function in vivo is still unclear [10]. Heat shock protein 23 (hsp23) was up-regulated by 2.03 and 9.33-fold while heat shock protein cognate 4 (hsc4) was down-regulated by $65 \%$. Heat shock proteins function as important chaperones intra-cellularly for other proteins in protein-protein interactions such as folding and assisting in the establishment of proper protein conformation (shape) and prevention of unwanted protein aggregation. The heat shock cognate genes play critical roles in chaperoning of cellular proteins, and they are expressed in a more constitutive manner compared to the inducible heat shock genes [25]. Aldehyde dehydrogenase X, phosphoglycerate kinase, oxidoreductase TM_0325, superoxide dismutase [Mn], and nucleoside diphosphate kinase were down-regulated by $50-87 \%$ while triosephosphate isomerase, dehydrogenase/reductase SDR family member 11, and aminopeptidase W07G4.4 were upregulated by 2.79-7.03 fold. 60S acidic ribosomal protein P1 was down-regulated by $58 \%$. 
Table 1. Differentially expressed proteins between 3-day-old eggs and 1-day-old larvae of B. latifrons.

\begin{tabular}{|c|c|c|}
\hline Accession \# & Protein Names (Abbreviations) & Spot ID (L1/E3 Ratio) \\
\hline XP_018793231.1 & Maltase 2 (Mal-B2) & E3-98 (0.25), 104 (0.11), $121(0.28), 129$ (0.35), 149 (0.05), $157(0.44), 183(0.26)$ \\
\hline XP_018784870.1 & Transferrin (TF) & E3-105 (0.04), $112(0.24), 113(0.07), 116(0.12), 128(0.04)$ \\
\hline XP_018798053.1 & Vitellogenin-1 (VTG1) & E3-252 (0.26), $257(0.36), 282(0.15), 291(0.34), 293(0.09)$ \\
\hline XP_018787380.1 & Vitellogenin-1 (VTG1) & E3-267 (0.14), $268(0.07), 276(0.25), 277(0.12), 281(0.25), 307(0.17), 316(0.10)$ \\
\hline XP_018787290.1 & Vitellogenin-2 (VTG2) & E3-308 (0.17), $312(0.11), 313(0.39), 317(0.46), 322(0.39), 326(0.43), 327(0.19)$ \\
\hline XP_018791497.1 & Protein amalgam (Ama) & E3-366 (0.32) \\
\hline XP_011201883.1 & Peroxiredoxin 1 (PRDX1) & E3-564 (0.27) \\
\hline XP_018795769.1 & Peptidyl-prolyl cis-trans isomerase 6 (PPIL6) & E3-628 (0.46) \\
\hline XP 018788060.1 & Heat shock protein 23 (Hsp23) & E3-633 $(0.16), 640(0.12), 643(0.08), 646(0.19), 650(0.23)$ \\
\hline XP_018797702.1 & Peroxiredoxin-5, MC (PRDX5) & E3-685 (0.49) \\
\hline XP 018799095.1 & Heat shock protein 83 (Hsp83) & E3-709 (0.25) \\
\hline XP_018793742.1 & SCO-spondin (SSPO) & L1-130 (4.37) \\
\hline XP 018793741.1 & Uncharacterized protein LOC108971872 isoform X2 & L1-136 (5.89) \\
\hline XP_004526477.1 & Actin-2, muscle specific & L1-304 (3.82) \\
\hline XP 018799378.1 & Tropomyosin-2 (TPM2) & L1-365 (20.31), L-378 (7.12) \\
\hline XP_018802321.1 & L-lactate dehydrogenase (LDH) & L1-372 (2.67) \\
\hline XP_018783435.1 & Malate dehydrogenase, cytoplasmic (MDH) & L1-379 (2.62) \\
\hline XP_018795679.1 & Uncharacterized protein LOC108973107 isoform X1 & L1-395 (5.17) \\
\hline XP_018789491.1 & UDP-glucose 4-epimerase & L1-401 (5.13), 407 (6.18), 485 (2.01) \\
\hline XP_018787334.1 & Glyceraldehyde-3-phosphate dehydrogenase (G3PD) & L1-416 (4.45) \\
\hline XP_018804543.1 & Trehalose-phosphate phosphatase (TPP) & L1-419 (15.82) \\
\hline XP_004519608.1 & T-complex protein 1 subunit gamma (TCP1) & L1-426 (23.12) \\
\hline XP_018804039.1 & 3-hydroxyisobutyrate dehydrogenase (HIBADH) & L1-454 (2.14) \\
\hline $\mathrm{XP} 018804543.1$ & Trehalose-phosphate phosphatase B(TPP) & L1-551 (2.04) \\
\hline XP_018794827.1 & Uncharacterized protein LOC108972624 & L1-563 (3.30), 565 (26.86) \\
\hline $\mathrm{XP} 011210145.1$ & Uncharacterized protein LOC105230849 & L1-566 (6.57) \\
\hline XP_018793665.1 & Protein lethal (2) essential for life (L2EFL) & L1-571 (2.13) \\
\hline XP 018791794.1 & Filamin-A, isoform X2 (FLNA) & L1-59 (34.34) \\
\hline XP_018787727.1 & Hypothetical protein c0_g1_i1 (c0_g1_i1) & L1-674 (15.72) \\
\hline XP_018804351.1 & Muscle-specific protein 20 (MP20) & L1-679 (3.02) \\
\hline gi $|880832606|$ & Krueppel ous protein 1 & L1-699 (7.44) \\
\hline XP_018799091.1 & General odorant-binding protein 99a (GOBP99a) & L1-708 (2.01) \\
\hline XP_018788026.1 & Myophilin & L1-710 (12.10) \\
\hline XP_014094514.1 & Larval cuticle protein 5 (LCP5) & $\mathrm{L} 1-721(7.70)$ \\
\hline XP_018797071.1 & $60 \mathrm{~S}$ acidic ribosomal protein P2 (RpLP2) & L1-739 (3.64) \\
\hline XP_018803514.1 & 60S acidic ribosomal protein P1 (RpLP1) & L1-750 (3.90) \\
\hline
\end{tabular}




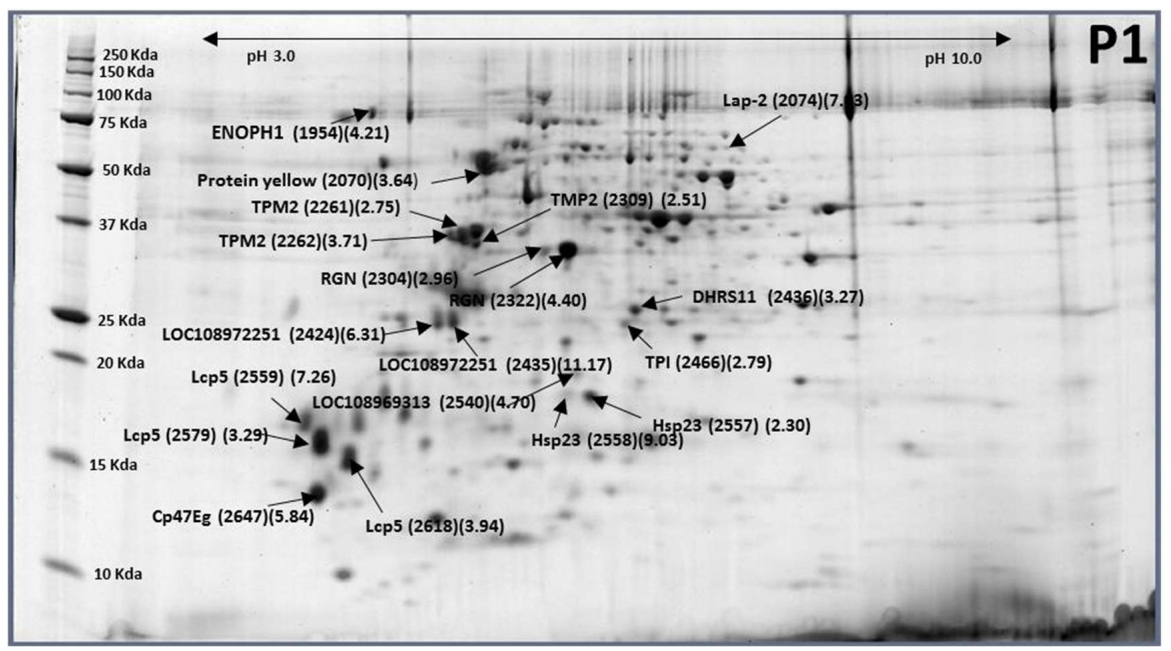

(A)

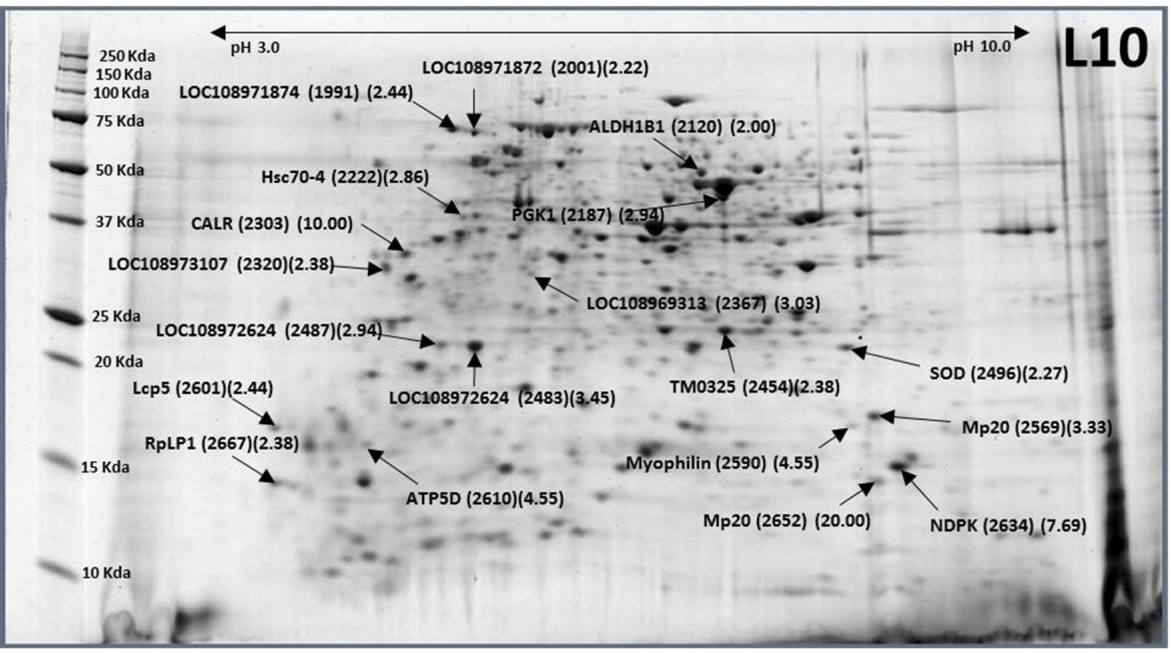

(B)

Figure 2. Representative images of 1-day-old pupae (P1) (A) and 10-day-old larvae (L10) (B) of Bactrocera latifrons. Images were labelled with protein names (spot ID\#) (P1/L10 ratios).

Table 2. Differentially expressed proteins between 10-day-old larvae and 1-day-old pupae of Bactrocera latifrons.

\begin{tabular}{lll}
\hline Accession No. & Protein Names (Abbreviations) & Spot ID (P1/L10 Ratio) \\
\hline XP_018793742.1 & Uncharacterized protein LOC108971874 & L10-1991 (0.41) \\
XP_018793741.1 & Uncharacterized protein LOC108971872 isoform X2 & L10-2001 (0.45) \\
XP_018783509.1 & Aldehyde dehydrogenase X (ALDH1B1) & L10-2120 (0.50) \\
XP_018790778.1 & Phosphoglycerate kinase (PGK1) & L10-2187 (0.34) \\
XP_011208284.1 & Heat shock protein cognate 4 (Hsc70-4) & L10-2222 (0.35) \\
XP_018794703.1 & Calreticulin (CALR) & L10-2303 (0.10) \\
XP_018795679.1 & Uncharacterized protein LOC108973107 isoform X1 & L10-2320 (0.42) \\
XP_018789492.1 & Uncharacterized protein LOC108969313 & L10-2367 (0.33) \\
XP_018802393.1 & putative oxidoreductase TM_0325 (TM0325) & L10-2454 (0.42) \\
XP_018794827.1 & Uncharacterized protein LOC108972625 & L10-2483 (0.29), L10-2487 (0.34) \\
XP_018788990.1 & Superoxide dismutase [Mn] (SOD) & L10-2496 (0.44) \\
XP_018804351.1 & Muscle-specific protein 20 (Mp20) & L10-2569 (0.30), L10-2652 (0.05) \\
XP_018788026.1 & Myophilin & L10-2590 (0.22) \\
gi| 880854006I & Larval cuticle protein 5 (Lcp5) & L10-2601 (0.41) \\
XP_018804878.1 & ATP synthase subunit delta (ATP5D) & L10-2610 (0.22) \\
\hline
\end{tabular}


Table 2. Cont.

\begin{tabular}{lll}
\hline Accession No. & Protein Names (Abbreviations) & Spot ID (P1/L10 Ratio) \\
\hline XP_004517938.2 & Nucleoside diphosphate kinase (NDPK) & L10-2634 (0.13) \\
XP_018803514.1 & 60S acidic ribosomal protein P1 (RpLP1) & L10-2667(0.42) \\
XP_018800124.1 & Enolase-phosphatase E1 & P1-1954 (4.21) \\
XP_018789027.1 & Protein yellow & P1-2070 (3.64) \\
XP_018802734.1 & Putative aminopeptidase W07G4.4 (lap-2) & P1-2074 (7.03) \\
XP_018799378.1 & Tropomyosin-2 (TPM2) & P1-2261 (2.75), P1-2262 (3.71) \\
XP_018802440.1 & Regucalcin (RGN) & P1-2304 (2.96), P1-2322 (4.40) \\
XP_018799300.1 & Tropomyosin-2 (TPM2) & P1-2309 (2.51) \\
XP_018794340.1 & Uncharacterized protein LOC108972251 & P1-2424 (6.31), P1-2435 (11.17) \\
XP_018786140.1 & Dehydrogenase/reductase SDR family member 11 (DHRS11) & P1-2436 (3.27) \\
XP_018793665.1 & Triosephosphate isomerase (TPI) & P1-2466 (2.79) \\
XP_018789492.1 & Uncharacterized protein LOC108969313 & P1-2540 (4.70) \\
XP_018788064.1 & Heat shock protein 23 (Hsp23) & P1-2557 (2.30), P1-2558 (9.03) \\
XP_018805087.1 & Larval cuticle protein 5 (Lcp5) & P1-2559 (7.26) \\
XP_018793318.1 & Larval cuticle protein 5 (Lcp5) & P1-2579 (3.29) \\
XP_018793320.1 & Larval cuticle protein 5 (Lcp5) & P1-2618 (3.94) \\
XP_018805010.1 & Cuticular protein 47Eg (Cp47Eg) & P1-2647 (5.84) \\
\hline
\end{tabular}

\subsection{Pupae (12-Day-Old, P12) to Females (1-Day-Old, F1)}

Major differentially expressed proteins between 12-day-old pupae and 1-day-old females were 3 down-regulated proteins (5 spots)—Reticulon-4-interacting protein 1 (RTN4Ip1), cuticle protein (CP), catalase, and 13 up-regulated protein spots containing 10 proteins-Dihydrolipoyl dehydrogenase (DLD), enolase, arginine kinase (AK), glyceraldehyde-3-phosphate dehydrogenase (GAPDH), pyruvate dehydrogenase $\mathrm{E} 1$ component subunit beta (PDHB), epidermal retinol dehydrogenase 2 (RDHE2), heat shock protein beta-1 (HSPB1), pericentrin (PCNT), endocuticle structural glycoprotein ABD-4 (CUD4-1), and general odorant-binding protein 99a (Table 3; Figure 3). Reticulon-4-interacting protein 1 (RTN4Ip1) plays a role in the regulation of retinal ganglion cell (RGC) neurite outgrowth, and hence in the development of the inner retina and optic nerve or dendrite development. All proteins involved in glycolysis (DLD, AK, GAPDH, PDHB) were upregulated. Epidermal retinol dehydrogenase 2 (RDHE2) is involved in the pathway retinol metabolism, which is part of cofactor metabolism. Heat shock protein beta-1 (HSPB1) functions as a molecular chaperone. It maintains denatured proteins in a folding-competent state and plays a role in stress resistance and actin organization. It may regulate numerous biological processes through its molecular chaperone activity including the phosphorylation and the axonal transport of neurofilament proteins. Pericentrin is an integral component of the centrosome. The centrosome serves as a multifunctional scaffold for anchoring numerous proteins and protein complexes. Endocuticle structural glycoprotein ABD-4 (CUD4_1) plays a role in cell-cell interaction.

Table 3. Differentially expressed proteins between 1-day-old females (F1) (3A) and 12-day-old pupae (P12) (3B) of Bactrocera latifrons.

\begin{tabular}{lll}
\hline Accession No. & Protein Name & Spot ID (F1/P12) \\
\hline XP_018798316.1 & Catalase & P12-211 (0.30) \\
XP_018794196.1 & Reticulon-4-interacting protein 1(RTN41PI) & P12-500 (0.03) \\
XP_018794197.1 & Cuticle protein (CP) & P12-519 (0.07) \\
XP_018794197.1 & Larval cuticle protein A2B & P12-522 (0.04) \\
XP_018788732.1 & Cuticle protein 8 (CP8) & P12-723 (0.10) \\
XP_018803223.1 & Dihydrolipoyl dehydrogenase (DLD) & F1-196 (2.53) \\
XP_018794316.1 & Enolase & F1-223 (2.03), F1-226 (2.48) \\
XP_018785511.1 & Arginine kinase (AK) & F1-299 (2.12) \\
XP_018804575.1 & Glyceraldehyde-3-phosphate dehydrogenase (GAPDH) & F1-363 (3.54) \\
XP_018798823.1 & Pyruvate dehydrogenase E1 component subunit $\beta$ (PDHB) & F1-403 (2.22) \\
XP_018801988.1 & Epidermal retinol dehydrogenase 2 (RDHE2) & F1-533 (2.60) \\
XP_018800458.1 & Heat shock protein beta-1 (HSPB1) & F1-541 (2.60) \\
XP_018801988.1 & Pericentrin (PCNT) & F1-548 (5.64) \\
XP_004520585.1 & Endocuticle structural glycoprotein ABD-4 (CUD-4) & F1-625 (2.00), F1-654 (3.12) \\
XP_018799095.1 & General odorant-binding protein 99a-like (OBP99a) & F1-632 (2.32) \\
XP_018787334.1 & Glyceraldehyde-3-phosphate dehydrogenase (GAPDH) & F1-779 (2.12) \\
\hline
\end{tabular}




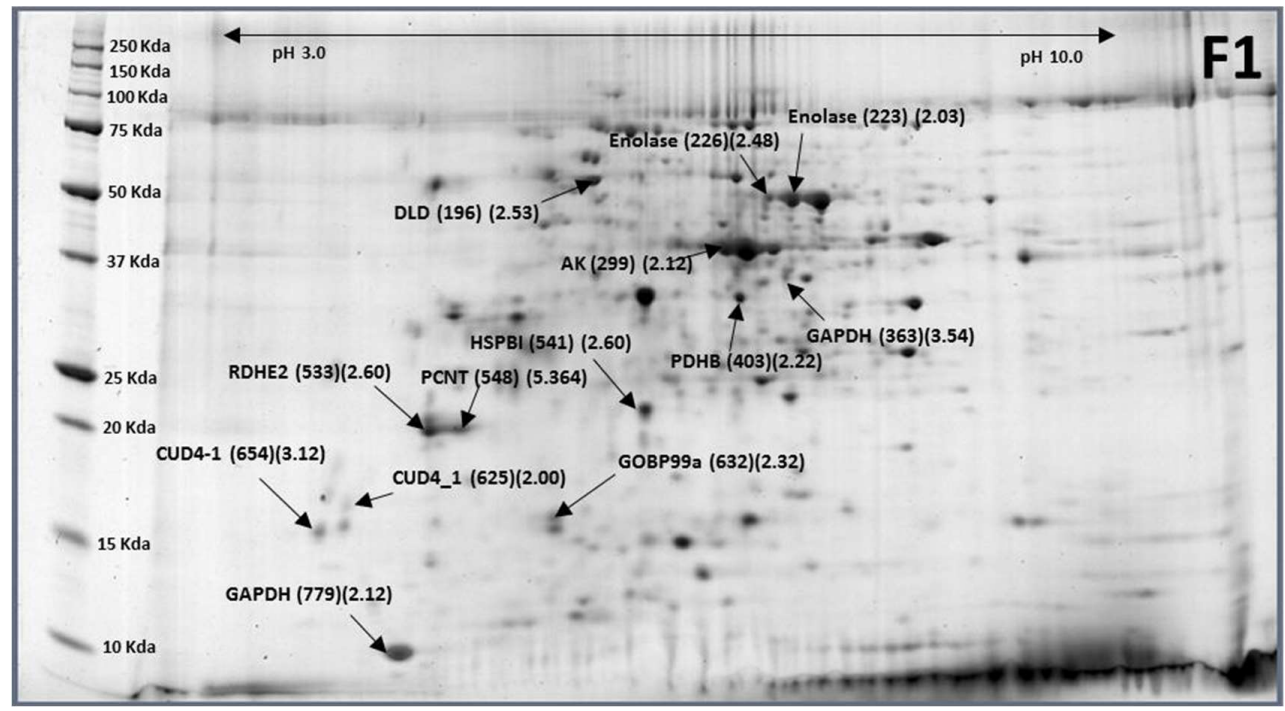

(A)

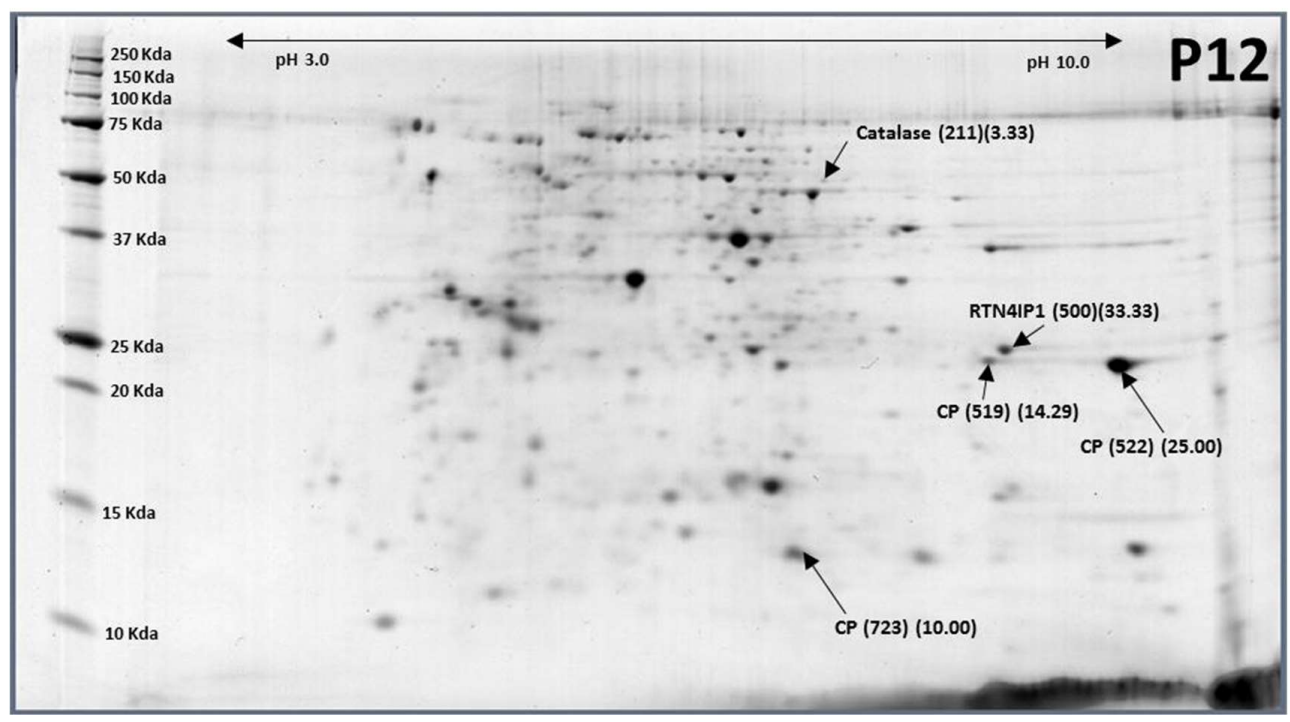

(B)

Figure 3. Representative images of 1-day-old females (F1) (A) and 12-day-old pupae (P12) (B) of Bactrocera latifrons. Images were labelled with protein names (spot ID\#) (F1/P12 ratios).

\subsection{Pupae (12-Day-Old, P12) to Males (1-Day-Old, M1)}

Major differentially expressed protein between 12-day-old pupae and 1-day-old males were six down-regulated protein spots containing four cuticle proteins and six up-regulated protein spots including six proteins-DLD, enolase, Heat shock protein cognate four (Hsc70-4), Arginine kinase (AK), DNA polymerase theta (Pol theta), and endocuticle structural glycoprotein ABD-4 (Table 4; Figure 4). Cuticle proteins were reduced by $89-97 \%(\mathrm{M} 1 / \mathrm{P} 12$ ratio $=0.03-0.11)$. Glycolysis proteins such as enolase, DLD, and AK were upregulated to restore from hibernated pupae to adult stage. Enolase (phosphopyruvate hydratase) is a metalloenzyme responsible for the catalysis of the conversion of 2-phosphoglycerate (2-PG) to phosphoenolpyruvate (PEP) in the ninth and penultimate step of glycolysis. AK can maintain ATP levels by the phosphorylation of the so-called "phosphagens", to rapidly replenish ATP. The function of Hsc70-4 is chaperone-binding/unfold protein-binding. Overexpression of DNA polymerase theta (Pol theta) in Drosophila melanogaster causes reduced hatch 
rate, and sensitivity to nitrogen mustard was reported. Polymerase theta (Pol theta) levels are important for genomic stability.

From pupal stage to adults, five proteins were identical between males and females. They were cuticle proteins (XP_018788732.1), dihydrolipoyl dehydrogenase (XP_018803223.1), enolase (XP_018794316.1), arginine kinase (XP_018785511.1), and endocuticle structural glycoprotein (XP_004520585.1). However, the differentially expressed proteins in females are more than in males.

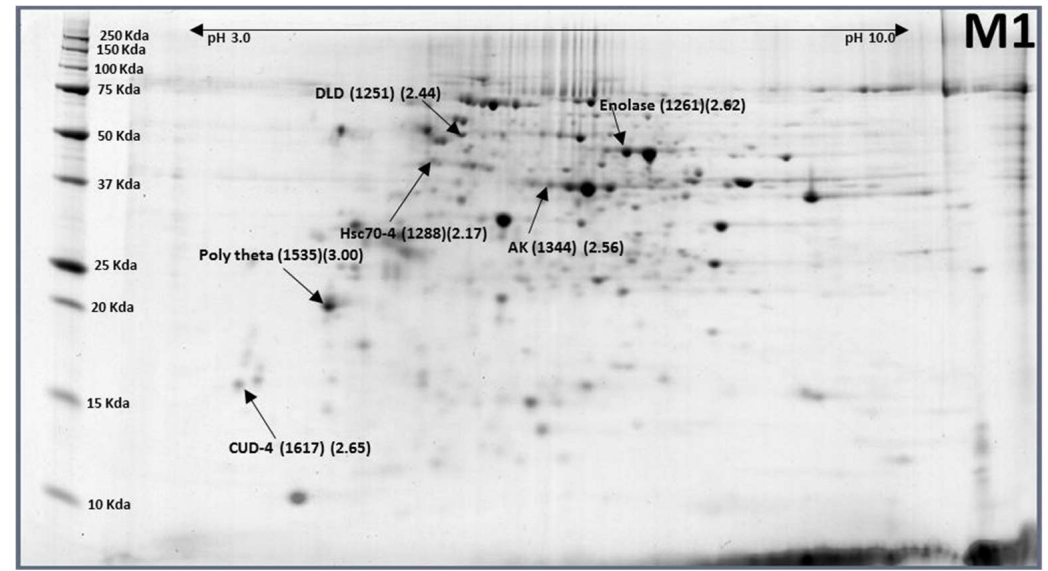

(A)

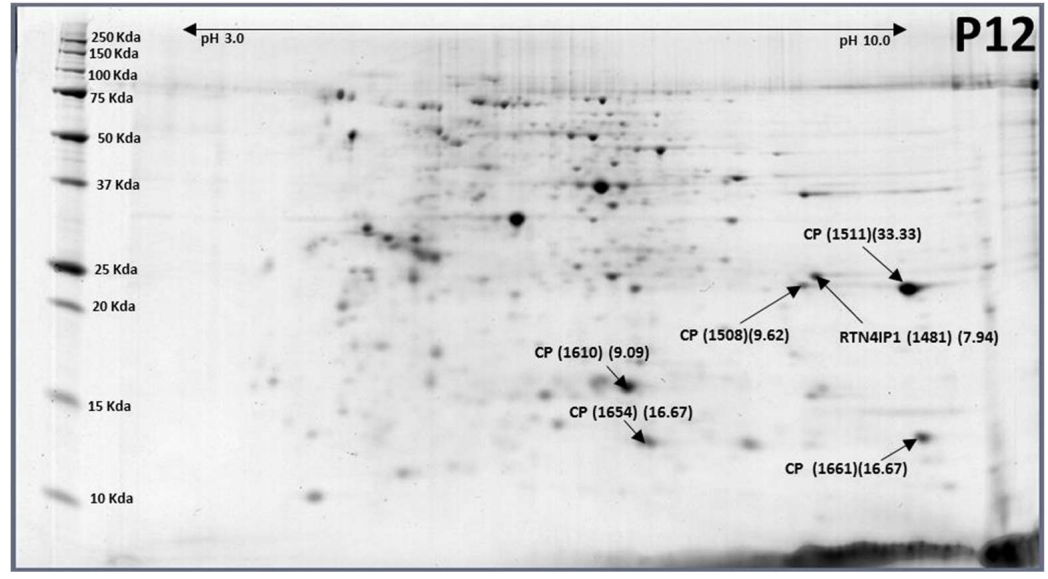

(B)

Figure 4. Representative images of 1-day-old males (A) and 12-day-old pupae (B) of Bactrocera latifrons. Images were labelled with protein names (spot ID\#) (M1/P12 ratios).

Table 4. Differentially expressed proteins between 12-day-old pupae and 1-day-old males of Bactrocera latifrons.

\begin{tabular}{lll}
\hline Accession No. & Protein Name & Spot ID (M1/P12) \\
\hline XP_018794197.1 & Cuticle protein (CP) & P12-1511 (0.03) \\
XP_018783307.1 & Cuticle protein (CP) & P12-1610 (0.11) \\
XP_018783299.1 & Cuticle protein (CP) & P12-1654 (0.06) \\
XP_018788732.1 & Cuticle protein 8 (CP8) & P12-1661 (0.06) \\
XP_018803223.1 & Dihydrolipoyl dehydrogenase (DLD) & M1-1251 (2.44) \\
XP_018794316.1 & Enolase & M1-1261 (2.62) \\
XP_014087797.1 & Heat shock protein cognate 4 (Hsp70-4) & M1-1288 (2.17) \\
XP_018785511.1 & Arginine kinase (AK) & M1-1344 (2.56) \\
XP_018801988.1 & DNA polymerase $\theta$ (Poly theta) & M1-1535 (3.00) \\
XP_004520585.1 & Endocuticle structural glycoprotein ABD-4 (CUD-4) & M1-1617 (2.65) \\
\hline
\end{tabular}




\subsection{Females (1-Day-Old)-Males (1-Day-Old)}

Only one protein spot expressed significantly differently between 1-day-old females and males (Figure 5). That was OBP56d. The same protein was expressed at pupal stage [26].

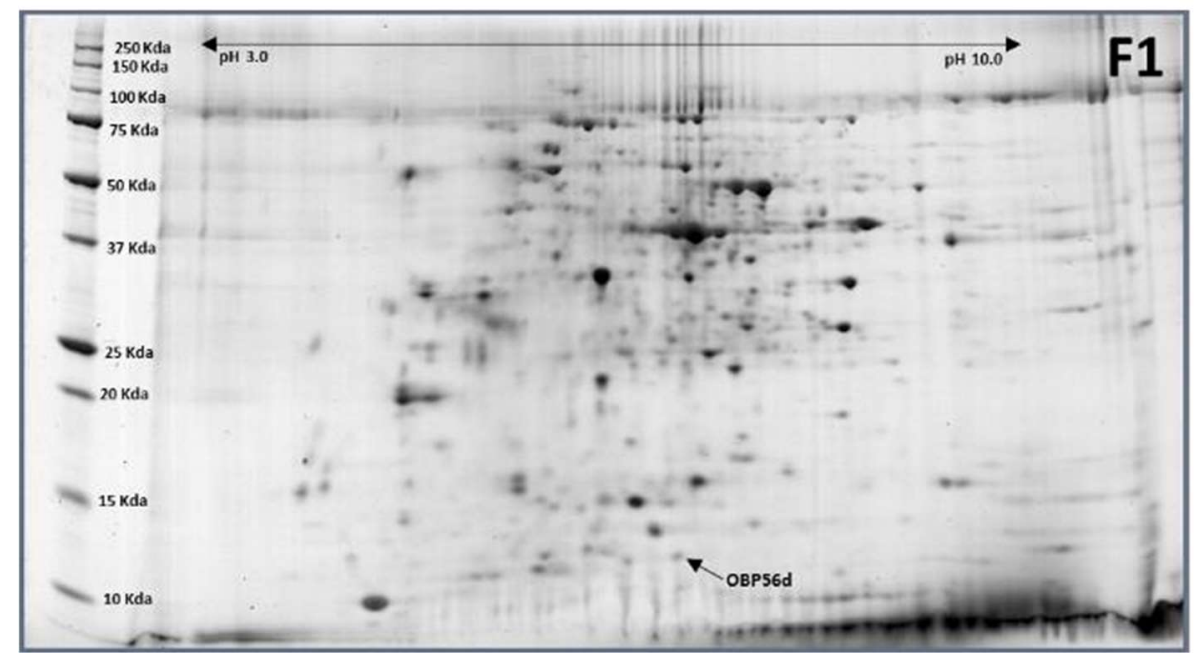

(A)

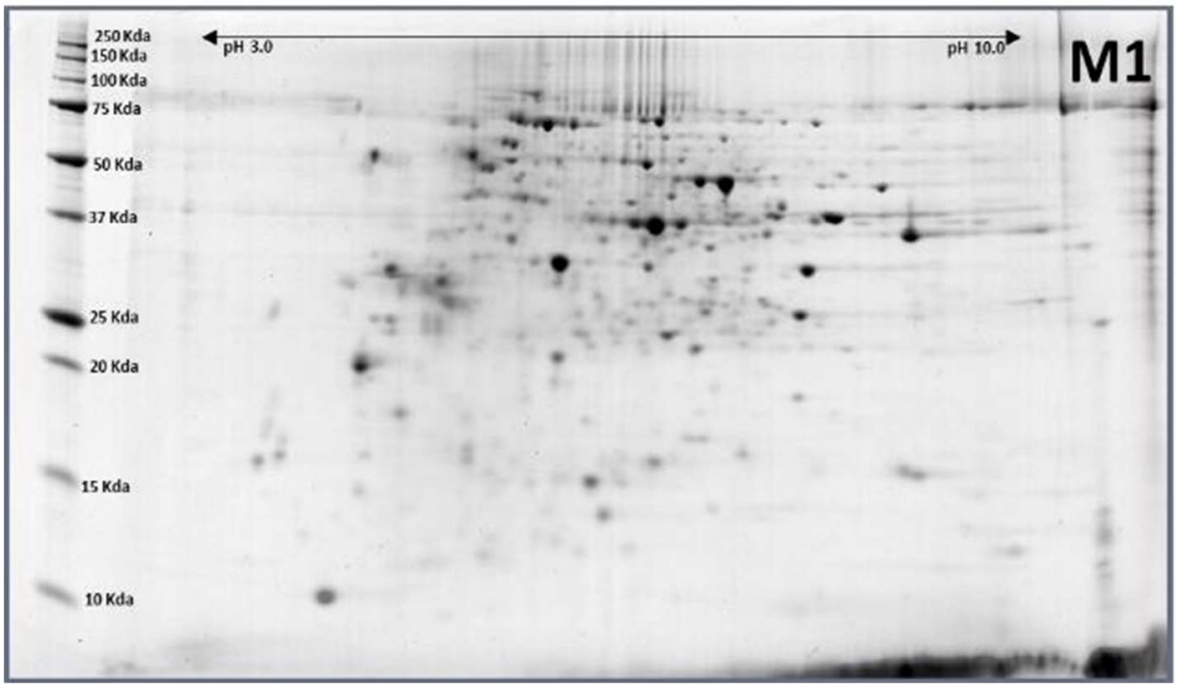

(B)

Figure 5. Representative images of 1-day-old females (F1) (A) and 1-day-old males (M1). (B) of Bactrocera latifrons. Images were labelled with protein names (spot ID\#) (F1/M1 ratios).

\subsection{Females (9-Day-Old)-Males (9-Day-Old)}

There were 15 differentially expressed protein spots between nine-day-old females and males. Four down-regulated protein spots contained four proteins, and 11 up-regulated protein spots contained eight proteins (Table 5 and Figure 6). Females significantly and differentially expressed vitellogenin- 1 by $>120$ fold over males. 


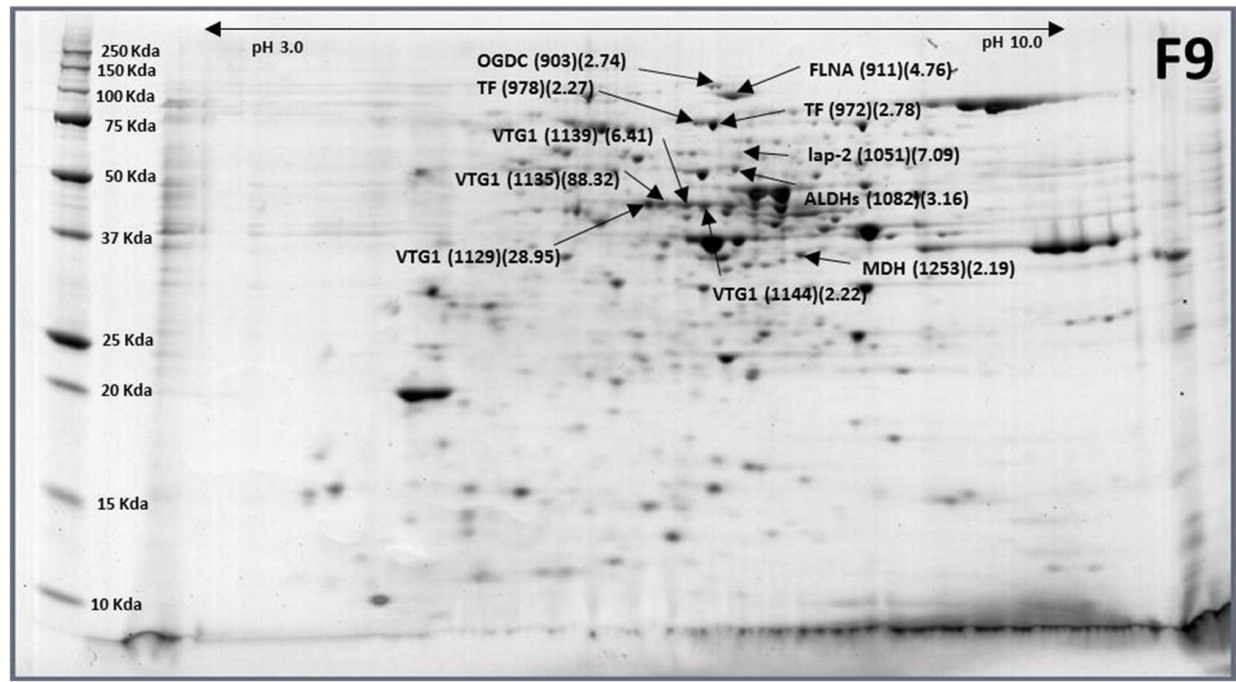

(A)

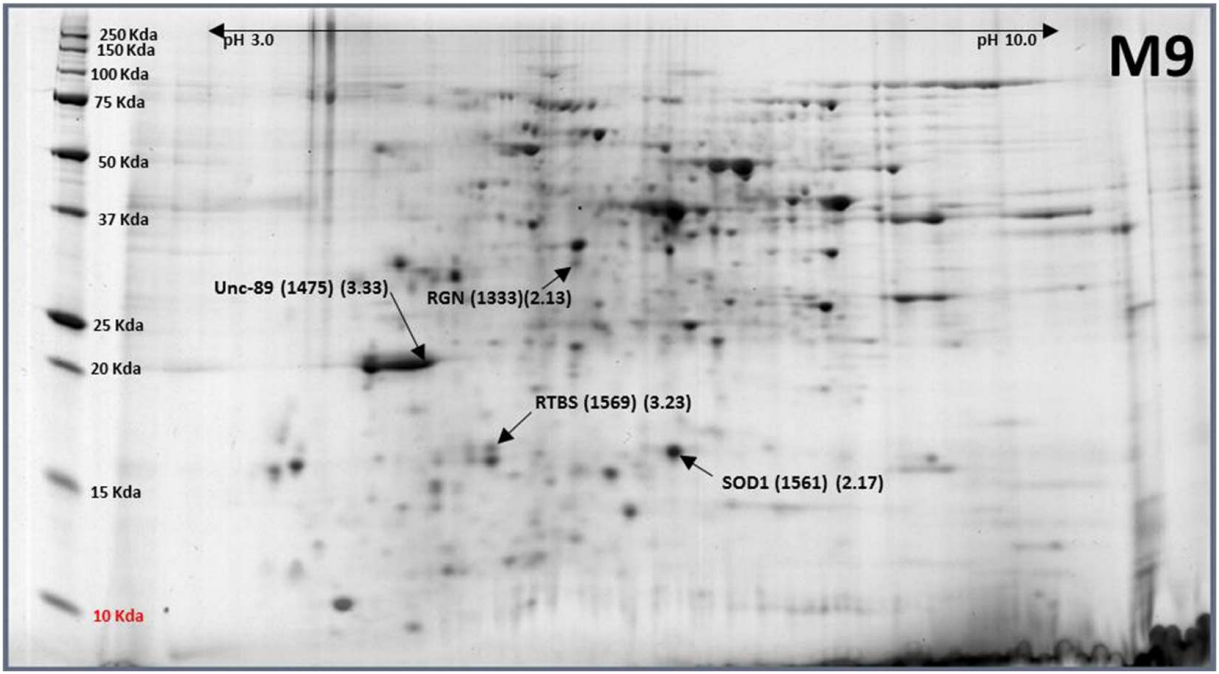

(B)

Figure 6. Representative images of 9-day-old females (A) and 9-day-old males (B) of Bactrocera latifrons. Images were labelled with protein names (spot ID\#) (F9/M9 ratios).

Table 5. Differentially expressed proteins between 9-day-old females and males of B. latifrons.

\begin{tabular}{lll}
\hline Accession No. & Protein Name & Spot ID (F9/M9 Ratio) \\
\hline XP_018804032.1 & Regucalcin (RGN) & M9-1333 (0.47) \\
XP_018801988.1 & Muscle M-line assembly protein unc-89 (UNC-89) & M9-1475 (0.30) \\
NP_001291905.1 & Superoxide dismutase [Cu-Zn] (SOD1) & M9-1561 (0.46) \\
XP_018799095.1 & RNA-directed DNA polymerase from transposon BS & M9-1569 (0.31) \\
XP_018802735.1 & (RTBS) & F9-1051 (7.09) \\
XP_018784524.1 & Putative aminopeptidase W07G4.4 (LAP-2) & F9-1082 (3.16) \\
XP_018787380.1 & Vitellogenin-1 (VTG1) & F9-1129 (28.95), F9-1135 (88.32), \\
& & F9-1139 (6.41), F9-1144 (2.22) \\
XP_018783435.1 & Malate dehydrogenase, cytoplasmic (MDH) & F9-1253 (2.19) \\
XP_018793355.1 & 2-oxoglutarate dehydrogenase (OGDC) & F9-903 (2.74) \\
XP_018791794.1 & Filamin-A (FLNA) & F9-911 (4.76) \\
XP_018784870.1 & Transferrin (TF) & F9-972 (2.78), F9-978 (2.27) \\
\hline
\end{tabular}




\section{Materials and Methods}

We established a proteomic profile between sequential developmental stages of the solanum fruit fly using 2D gel electrophoresis coupled with mass spectrometry for protein identification. Database searches were performed with Matrix Science's Mascot search engine v. 2.4 (www.matrixscience.com) on an in-house server against a concatenation of NCBI nr Insecta database, combined with a B. latifrons species-specific peptide database, derived from NCBI TSA BioProject: PRJNA281765. Differentially expressed proteins were compared and identified between each of two consecutive developmental stages and putative protein functions were annotated.

\subsection{Insects}

The B. latifrons colony collection was obtained initially from the USDA-ARS rearing facility in Hilo, HI, USA. Samples of varying ages of eggs ( 3 days), larvae ( 1 and 10 days), pupae (1 and 12 days), males ( 1 and 9 days), and females ( 1 and 9 days) were appropriately collected and stored at $80^{\circ} \mathrm{C}$ for protein analysis process.

\subsection{Protein Extraction}

We followed our standard proteomic protocols for sample preparation, 2D electrophoresis, and MS/MS (mass spectrometry) analysis [26]. Samples from all stage samples (0.25 g adults or pupae, $0.7 \mathrm{~g}$ eggs or larvae/mL buffer), were homogenized in one $\mathrm{mL} 10 \mathrm{mM}$ Tris- $\mathrm{HCl}$ (pH 7.0) containing protease inhibitors (final dilution =1:100; Sigma, St. Louis, MO, USA) for $15 \mathrm{~s}$ three times, with $30 \mathrm{~s}$ intervals using a Fast Prep-24 Instrument (MP Biomedicals, Solon, OH, USA). Homogenates were centrifuged twice at $15,294 \mathrm{~g}$ for $15 \mathrm{mins}$ at $4^{\circ} \mathrm{C}$. The resulting infranatants were transferred to new vials on ice for immediate use.

\subsection{Protein Quantification}

Total proteins were quantified using the Pierce Micro BCA Protein Assay Kit, using BSA as a quantitative standard (Thermo Scientific, Rockford, IL, USA). Three independent biological replicates from the same generation were processed for each treatment.

\subsection{D-Electrophoresis}

\subsubsection{IEF (Isoelectric Focusing): Separating Different Proteins Based on $\mathrm{pH}$ Value}

Five microliters of 2D gel protein standards (Bio-Rad, Hercules, CA, USA, \#161-0320) were added to each sample tube. IPG strips (pH 3-10; $11 \mathrm{~cm}$; Bio-Rad, \#163-2014) were rehydrated overnight with these sample solutions. Iso-electric focusing was performed with a Protean IEF cell system (Bio-Rad, Hercules, CA, USA) using the standard protocol and a preset linear volt ramp program (8000 V and $50 \mu \mathrm{A} /$ strip max., $35,000 \mathrm{vH})$. The focused strips were stored at $-80^{\circ} \mathrm{C}$ for later use.

\subsubsection{SDS Gel Electrophoresis Based on Molecular Weight (MW)}

The IPG strips were equilibrated (15 min/buffer: $6 \mathrm{M}$ urea, 2\% SDS, 20\% glycerol, $130 \mathrm{mM}$ DTT, $0.375 \mathrm{M}$ Tris-HCl, pH 8.7 [Buffer I] followed by $6 \mathrm{M}$ urea, 2\% SDS, 20\% glycerol, $135 \mathrm{mM}$ iodoacetamide, $0.375 \mathrm{M}$ Tris-HCl, and pH 8.7 [Buffer II]) for the second dimension. Prior to running, molecular weight standards were applied $(10 \mu \mathrm{L} /$ lane, Bio-Rad \#161-applied to each gel (precast gels, 8-16\% Tris-HCl, Bio-Rad \#345-0105), and proteins were separated on SDS-PAGE using the Criterion Cell system (Bio-Rad, Hercules, CA, USA, \#165-6001). Gels were stained with Coomassie Blue G-250 (BioSafe Stain, Bio-Rad, Hercules, CA, USA) and scanned using densitometer (Bio-Rad GS-900), and the images were analyzed using Delta 2D software (Decodon GmbH, Greifswald, Germany). Protein spots with ratios (either larger than two or smaller than 0.5) significantly different between treatments at $95 \%$ or above significant level using Students' $t$-test, were removed from the gels using a $1.5 \mathrm{~mm}$ 
spot picker (The Gel Company, San Francisco, CA, USA) and stored at $-80^{\circ} \mathrm{C}$ trypsin digestion and MS/MS analysis. Three independent biological replicates were performed.

\subsection{MS/MS Analysis: Identification of Protein Identity}

In preparation for MS/MS analysis, proteins were digested with trypsin, extracted, and then lyophilized and reconstituted with water [27]. A portion of each protein was mixed with alpha-cyano-4-hydroxycinnamic acid matrix and applied to the MALDI target and analyzed. The resulting sequence data (ratios, standard errors, $p$ values), combined with observed protein name, MW and $\mathrm{pI}$ values, protein score, ion score, protein \%, E value, and peptide sequence (with highest ion score) were used to establish protein identities (Supplementary file 1).

\subsection{Data Search and Analysis}

Database searches were performed with Matrix Science's Mascot search engine v. 2.4 (www.matrixscience.com) on an in-house server against the updated NCBInr "Blat 20160224" database and the NCBInr Insects database. The custom B. latifrons peptide database, generated from a draft annotation set of the $B$. latifrons genome is available as supplemental information (Supplementary files 2 and 3, NCBI BioProject 281765).

\section{Conclusions}

In summary, we found: 10 downregulated and 24 upregulated proteins between eggs and larvae, 17 downregulated and 12 upregulated proteins between larvae and pupae, 3 downregulated and 10 upregulated proteins between pupae and females, 4 downregulated and six upregulated proteins between pupae and males, and one was identified as significantly differentially expressed between 1-day-old females and males, while 4 downregulated and 8 upregulated proteins were identified between 9-day-old females and males. Proteins that expressed significantly differentially in both L1/E3 and P1/L10 are SCO-spondin (SSPO), hypothetical protein LOC108971872 isoform X2, LOC108973107 isoform X1, LOC108972624, muscle-specific protein 20 (msp20), myophilin, larval cuticle protein 5, $60 \mathrm{~S}$ acidic ribosomal protein P1, and tropomyosin-2. Glyceraldeyde-3-phosphate dehydrogenase is the only protein differentially expressed at both L1/E3 and F1/P12. Heat shock protein cognate 4 is the only protein differentially expressed at both P1/L10 and F1/P12. At both F1/P12 and M1/P12, 5 proteins (cuticle protein, dihydrolipoyl dehydrogenase, enolase, arginine kinase, and endocuticle structural glycoprotein ABD-4 (CUD4_1) are differentially expressed. Actin has been reported by in Ceratitis capitata, the "Mediterranean fruit fly" or "Medfly" [1]. They found that this gene was presented in the Medfly during late larval and late pupal development as well as in thoracic and leg tissue preparations from newly emerged adults. However, we found the Actin gene significantly differentially expressed in the early stage of B. latifrons larvae. The pupal stage is the stage that produced the most significantly differentially expressed proteins.

Understanding fruit fly basic biology in the development of each life stage in their life cycle at molecular level has been the most important element for improving the quality of mass-rearing fruit flies for SIT. We have profiled the proteome analysis of Bactrocera latifrons development via proteomics approach. From this study, we learned "what" these differentially expressed proteins are within each developmental stage and between each of two subsequent developmental stages, and when and where they express. This information will be used as a foundational/baseline information to later manipulate or identify the causes/pathway from any biotic or abiotic influences to explain why and how they express using gene editing techniques. This study will supply a wealth of additional information on understanding fruit fly development at molecular level. Profiling all four fruit fly species in Hawaii, which represents three important genera, has been ongoing.

Supplementary Materials: Supplementary materials can be found at http:/ /www.mdpi.com/1422-0067/19/7/ 1996/s1. 
Author Contributions: C.L.C. conceived and designed the experiments; C.L.C. performed the experiments; C.L.C. and S.G. analyzed the data; C.L.C. wrote the paper.

Funding: This research received no external funding.

Acknowledgments: The authors thank Beverly DaGue, M.U. Protein Center, for her expert work on mass spectrometry and protein analysis presented in this report. We also thank David Stanley for his guidance, and Cindy Goodman for her assistance to deliver the samples for MS/MS analysis. We would like to extend our gratitude to MaryAnn Villalun, Briette Corpuz, Amy Olsen and Thomas Ramangmou for helping to collect experimental data, analyzing and identifying proteins using Delta 2D software. Mention of trade names or commercial products in this article is solely for the purpose of providing specific information and does not imply recommendation or endorsement by the U.S. Department of Agriculture. All programs and services of the U.S. Department of Agriculture are offered on a nondiscriminatory basis without regard to race, color, national origin, religion, sex, age, marital status, or handicap.

Conflicts of Interest: The authors have declared no conflict of interest.

\section{References}

1. Shashank, P.R.; Haritha, B. Insect proteomics: Present and future prospective. Curr. Biotica 2014, 7, 336-342.

2. Vargas, R.I.; Nishida, T. Life history and demographic parameters of Dacus latifrons (Diptera: Tephritidae). J. Econ. Entomol. 1985, 78, 1242-1244. [CrossRef]

3. Liquido, N.J.; Harris, E.J.; Dekker, L.A. Ecology of Bactrocera latifrons (Diptera:Tephritidade) populations: Host plants, natural enemies, distribution, and abundance. Ann. Entomol. Soc. Am. 1994, 87, 71-84. [CrossRef]

4. McQuate, G.T.; Liquido, N.J. Annotated world bibliography of host fruits of Bactrocera latifrons (Hendel) (Diptera: Tephritidae). Insecta Mundi 2013, 289, 1-61.

5. Vargas, R.I.; Walsh, W.A.; Kanehisa, D.; Jang, E.B.; Armstrong, J.W. Demography of four Hawaiian fruit flies (Diptera: Tephritidae) reared at five constant temperatures. Ann. Entomol. Soc. Am. 1997, 90, $162-168$. [CrossRef]

6. Seeger, M.A.; Haffley, L.; Kaufman, T.C. Characterization of amalgam: A member of the immunoglobulin superfamily from Drosophila. Cell 1988, 55, 589-600. [CrossRef]

7. Zeev-Ben-Mordehai, T.; Mylonas, E.; Paz, A.; Peleg, Y.; Toker, L.; Silman, I.; Svergun, D.I.; Sussman, J.L. The quaternary structure of amalgam, a Drosophila neuronal adhesion protein, explains its dual adhesion properties. Biophys. J. 2009, 97, 2316-2326. [CrossRef] [PubMed]

8. Khoshnood, B.; Dacklin, I.; Grabbe, C. Urm1: An essential regulator of JNK signaling and oxidative stress in Drosophila melanogaster. Cell. Mol. Life Sci. 2016, 73, 1939-1954. [CrossRef] [PubMed]

9. Theodoraki, M.A.; Mintzas, A.C. cDNA cloning, heat shock regulation and developmental expression of the hsp83 gene in the Mediterranean fruit fly Ceratitis capitata. Insect Mol. Biol. 2006, 15, 839-852. [CrossRef] [PubMed]

10. Rourke, I.J.; East, P.D. Evidence for gene conversion between tandemly duplicated cytoplasmic actin genes of Helicoverpa armigera (Lepidoptera:Noctuidae). J. Mol. Evol. 1997, 44, 169-177. [CrossRef] [PubMed]

11. Williams, J.; Boin, N.G.; Valera, J.M.; Johnson, A.N. Noncanonical roles for Tropomyosin during myogenesis. Development 2015, 142, 3440-3452. [CrossRef] [PubMed]

12. Chen, Q.; Haddad, G.G. Role of trehalose phosphate synthase and trehalose during hypoxia: From flies to mammals. J. Exp. Biol. 2004, 207, 3125-3129. [CrossRef] [PubMed]

13. Alcolea, P.J.; Alonso, A.; Larraga, V. Proteome profiling of Leishmania infantum promastigotes. J. Eukaryot. Microbiol. 2011, 58, 352-358. [CrossRef] [PubMed]

14. Bhaskar, M.K.; Kuldeep, J.; Siddiqi, M.I.; Goyal, N. The TCP1 $\gamma$ subunit of Leishmania donovani forms a biologically active homo-oligomeric complex. FEBS J. 2015, 282, 4607-4619. [CrossRef] [PubMed]

15. Jagla, T.; Wojtowicz, I.; Jagla, K. Lethal (2) Essential for Life Gene Regulates Lateral Muscle Shapes during Embryonic Development; GReD: Clermont, France, 2014.

16. Kurzik-Dumke, U.; Lohmann, E. Sequence of the new Drosophila melanogaster small heat-shock-related gene, lethal(2) essential for life [1(2)efl], at locus 59F4,5. Gene 1995, 154, 171-175. [CrossRef]

17. Feng, Y.; Walsh, C.A. The many faces of filamin: A versatile molecular scaffold for cell motility and signalling. Nat. Cell Biol. 2004, 6, 1034-1038. [CrossRef] [PubMed] 
18. Ohta, Y.; Hartwig, J.H.; Stossel, T.P. FilGAP, a Rho- and ROCK-regulated GAP for Rac binds filamin A to control actin remodelling. Nat. Cell Biol. 2006, 8, 803-814. [CrossRef] [PubMed]

19. Popowicz, G.M.; Schleicher, M.; Noegel, A.A.; Holak, T.A. Filamins: Promiscuous organizers of the cytoskeleton. Trends Biochem. Sci. 2006, 31, 411-419. [CrossRef] [PubMed]

20. Stossel, T.P.; Condeelis, J.; Cooley, L.; Hartwig, J.H.; Noegel, A.; Schleicher, M.; Shapiro, S.S. Filamins as integrators of cell mechanics and signaling. Nat. Rev. Mol. Cell Biol. 2001, 2, 138-145. [CrossRef] [PubMed]

21. Yue, J.G.; Huhn, S.; Shen, Z. Complex roles of filamin-A mediated cytoskeleton network in cancer progression. Cell Biosci. 2013, 3, 7. [CrossRef] [PubMed]

22. Ayme-Southgate, A.; Lasko, P.; French, C.; Pardue, M.L. Characterization of the gene for mp20: A Drosophila muscle protein that is not found in asynchronous oscillatory flight muscle. J. Cell Biol. 1989, 108, 521-531. [CrossRef] [PubMed]

23. Qiu, F.; Brendel, S.; Cunha, P.M.; Astola, N.; Song, B.; Furlong, E.E.; Leonard, K.R.; Bullard, B. Myofilin, a protein in the thick filaments of insect muscle. J. Cell. Sci. 2005, 118, 1527-1536. [CrossRef] [PubMed]

24. Vera, A.; Stanic, K.; Montecinos, H.; Torrejón, M.E.; Marcellini, S.; Caprile, T. SCO-spondin from embryonic cerebrospinal fluid is required for neurogenesis during early brain development. Front. Cell. Neurosci. 2013, 7, 80. [CrossRef] [PubMed]

25. Thanaphum, S.; Haymer, D.S. A member of the hsp70 gene family from the Mediterranean fruit fly, Ceratitis capitata. Insect Mol. Biol. 1998, 7, 63-72. [CrossRef] [PubMed]

26. Chang, C.L. Proteomic identification of a potential sex biomarker for 2 fruit fly species at pupal stage. J. Asia Pac. Entomol. 2017, 20, 125-131. [CrossRef]

27. Stanley, D.W.; Goodman, C.; An, S.; McIntosh, A.; Song, Q. Prostaglandins A1 and E1 influence gene expression in an established insect cell line (BCIRLHzAM1cells). Insect Biochem. Mol. Biol. 2008, 38, 275-284. [CrossRef] [PubMed]

(C) 2018 by the authors. Licensee MDPI, Basel, Switzerland. This article is an open access article distributed under the terms and conditions of the Creative Commons Attribution (CC BY) license (http:/ / creativecommons.org/licenses/by/4.0/). 